\title{
Water Quality Parameter Monitoring System in Multi-Region Based on IOT
}

\author{
Hui Li ${ }^{*}$ and Jing Li \\ Faculty of Automation, Huaiyin Institute of Technology, HuaiAn 223003, China \\ lijing_1981_312@163.com \\ *The corresponding author
}

Keywords: Monitor, Multi-Region, Internet of things, Cost effectiveness

\begin{abstract}
For multi-region fishery water environment, multi-regional water quality online monitoring system is present in this paper, which is implemented in the Android mobile phone. And through it many of water quality parameters in different water region can be monitored online, such as water level, salinity, $\mathrm{pH}$, flow rate, temperature, dissolved oxygen and so on. All these parameters will be saved in SQLite database in Android mobile memory. Communication parameters and alarm threshold parameters can be saved as ini file in TXT mode. The design adopts the mode of socket network communication based on message notification mechanism. The design has low requirement on the network, and has high cost effectiveness. After experimenting and debugging in Jiangsu province Liyang CHANGDANG lake experiment base, the indicators of the system response is good, easy to use, which provides the guidance of fishery production and increase the income of fishermen?
\end{abstract}

\section{Introduction}

Fishery development needs environmental with quality waters ${ }^{[1,2]}$. The system of Fishery water quality parameter monitoring and alarming in many water areas based on IOT provides security for the development of fishery. At present, the Internet of things technology at home started late, and most of monitoring mode of water quality parameters is manual mode, and the system has poor real-time performance and inconvenient operation ${ }^{[3-5]}$. Fishery water quality parameter multi-point monitoring and alarming based on IOT has not been reported. And the system adopts the technology of IOT and monitors fishery waters quality parameters on-line in the Android mobile phone the and alarms to the fisher if the parameters of the system was overrun. The system is simple in operation, quick reaction, good real-time performance, and provides reference for fishermen to understand fishery waters real-time.

\section{The Whole System Framework}

The system is composed of 8 wireless sensor network unit, the local server, the remote client and remote mobile phone user clients. System structure diagram is shown in Fig. 1.

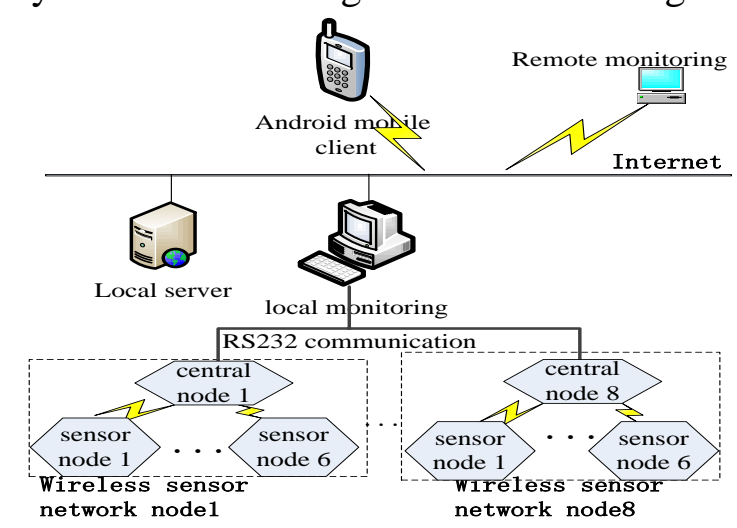

Figure 1. whole framework of system 


\section{Wireless Sensor Network Unit}

Water quality analog signal collected by sensor module would be transformed into digit signal after processing, and transmit to the central coordinating node through wireless communication module. Wireless sensor network unit is mainly responsible for collecting quality parameters from different 6 points in a water fields, and each point data contains 6 parameters (water level, salinity, $\mathrm{pH}$, flow rate, temperature, dissolved oxygen), after 8051CPU averaging processing through the serial port RS232 is sent to the monitoring computer, upload to internet. Each of the wireless sensor network units consists of 6 sensor nodes and a central coordinating node [11-12]. Central coordinating node and sensor nodes within the range covered adopt star network topology with the lowest energy. The processor uses SOC chip CC2430 and ZigBee protocol. The sensor node consists of wireless transceiver module and sensor module [13].

\section{Android Client Design}

Android is named from mobile phone operating system based on Linux platform announced by Google in November 5, 2007. It is composed of the operating system, middleware, user interface and application software and so on. The design is based on Android system, Lenovo A288t mobile phone, the system test version of Android2.3.5, kernel 2.6.35.7. The development environment of the system is Android SDK+JAVA and JDK6+Eclipse3.5. The system adopts the mode of client/server. The client uses Android JAVA development, storages the data using the database SQLite, and uses the SOCKET network widget to realize the network communication. The part of server is programmed by Delphi and SQL. Android client program is compiled in eclipse compiler environment and packed into the APK file. After setting the development authority in Android mobile phone users can install the APK file directly [6].

The system interface designed using XML script language, is composed of the login interface, the main monitoring interface and several water monitoring interface. Login interface is used for user's login, and the user's password is managed by database. the main monitoring interface is the main interface of the system, as shown in Fig. 1. Main monitoring interface is mainly responsible for all the water quality environment monitoring and alarm. There are 8 groups of state at the interface, each state with 1 light bulbs with 1 labels to show, if the water quality over the lamp lit at the same time, label show "alarm". Each state is arranged "view" button below the label, you will automatically jump to a water quality monitoring interface specific if you push the button.

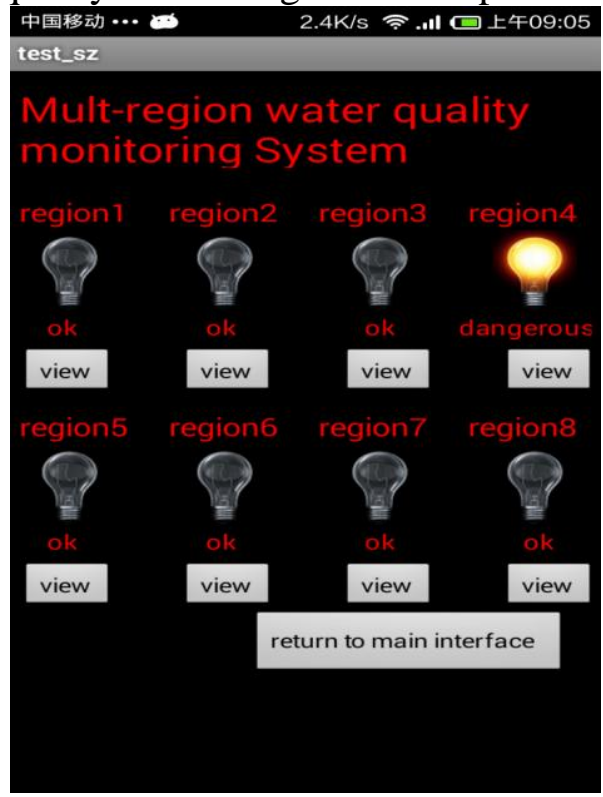

Figure 2. main monitoring and alarming interface

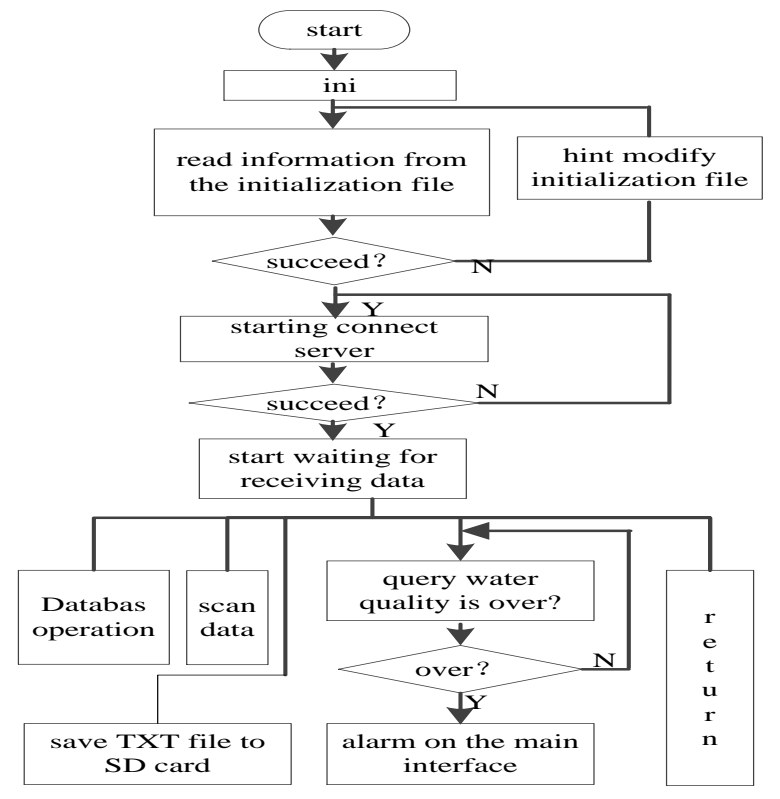

Figure 3. main flow chart of the system 
The main work flow as shown in Fig. 3. Firstly, system initialization, read information and communication parameters of each water alarm threshold information in the initialization file, and then connect to a server to start receiving one group data, assign to each area for the call display, compare the queried data and alarm threshold data in all the waters, and alarm when needed.

There are many design methods for alarming and communication parameters input module, can use the database method, also can use file method. Taking into account the design need simple and convenient, second kinds of design scheme is choosed.

The alarm threshold of water quality parameters and the communication parameters are set to initialization file in a fixed format, and stored in the SD card in TXT format. Before the first execution of the application, the user should open the initialization file(ini.txt) in the root directory, and modify the communication parameters according to a predetermined format (IP and port number) and 8 alarm upper and lower limit of water quality parameters (temperature, $\mathrm{pH}$, dissolved oxygen, salinity, water level, flow velocity).

Firstly Android system readed data from the initialize file by the style of data stream, and then parsed, and finally get useful data sending to the global variable of android.

The main methods are shown as follows:

String fileName = "/sdcard/ini.txt";

String tmp ="";

Try \{

FileInputStream ftmp = new FileInputStream (fileName);

Int length = ftmp.available (); Byte[] buffer_data = new byte[length]; ftmp.read (buffer_data);

tmp = EncodingUtils.getString (buffer_data, "GB2312"); String spStr[] = tmp.split (":|;");

String ip $=\operatorname{spStr}[1] ; \quad$ String port=spStr[3];

Finally, when the data is written into the SD card or the data is read from SD card, declaring the permission is obligatory in the manifest.xml configuration file, otherwise it is impossible to use.

\section{Database Module Design}

System uses SQLite database of the android, SQLite database is very little, and the retrieve efficiency of it is very high. There are two places using database in the system, the one is used when users logged in, to save the user name and password; another is when the system is running, to save the water quality parameters.

Android does not automatically provide database. When SQLite database is used in the Android application, the client must create the database firstly, and then create a table, index, filling data. So you should create a database firstly, and then create two tables. In Android there are two main ways to achieve access to the database, one is ADB shell, another is operate the database indirectly through the Android Java class. This design mainly uses the second way, using the abstract class SQLiteOpenHelper to create the database. According to the need of application development, The SQLiteOpenHelper class packages some logic function using to create and update the database. A subclass of SQLiteOpenHelper is composed of at least three method.Through the SQLiteDatabase class to implement function of inserting data, deleting data, querying data, modifing data and other operations in the database. When completed the operation of the database, system call Close () method in the SQLite Database to release the database connection.

\section{The Network Communication Module Design}

Communication module of the system mainly uses socket network communication. Socket is the cornerstone of communication, is a basic operation unit of network communication support TCP/IP protocol. Create an socket connection, you can specify the transport layer protocol, and socket supports two different transport layer protocols: TCP and UDP protocol. Considering the data security, this 
system design uses the TCP protocol, in order to improve the communication efficiency of the system, Socket takes a message mechanism to receive, ensuring rapid response.Mobile phone client read the data stream in the initialization file (ini.txt) firstly and then successfully isolated the IP address and port number, created a socket to the server sending a connection request, and then waited for the information form the server. After receiving the client connection requests, the server gets the client's IP address and port number, and then sends to the client the water quality parameters information timely. The specific format of receiving data: FA:1\# water temperature, $\mathrm{pH}$ value, water level, dissolved oxygen, salinity, waters flow,... 8\# water temperature, $\mathrm{pH}$ value, water level, dissolved oxygen, salinity, waters flow, FB.

\section{Summary}

The system is used in Jiangsu province china Liyang CHANGDANG lake crab experimental base 30 metres x 30 metres, real-time get the water quality parameter data of temperature, $\mathrm{pH}$, dissolved oxygen, salinity, water level, flow, and stored in the database. After long time using and testing the monitoring alarm system of, we can see that system operation is stable, not strike, the operation interface is flexible, convenient control, get rid of the influence of time, geographical environment and other factors. As long as the GPRS network signal is available the system can monitor simultaneously on multiple water on-line and automatic alarm. And the system needs low network signal, has the very high rate of capability to price. The system has been widely used in the project supported by the advantage subject construction engineering of colleges and universities in Jiangsu province. And the system has been also widely used in the project of Municipal Commission of Economy and Information Technology intelligent agriculture IOT application demonstration project. Thus the system has very strong popularization and practical value.

\section{Acknowledgement}

This research was financially supported by Huaian agricultural science and technology guidance project (HANZ2014007), Jiangsu Province 2013 Graduate Students Research and Innovation Program (CXLX13_669), Jiangsu Agricultural Science and Technology Support Project (BE2013402).

\section{References}

[1] Shi Bing, Zhao Dean, Liu Xinqiao, et al. Intelligent monitoring system for industrialized aquaculture based on wireless sensor network[J]. Transactions of the Chinese Society of Agricultural Engineering, 2011, 27(9):136-140

[2] Shi Bing, Zhao Dean, Liu Xingqiao, et al. Design of Intelligent Monitoring System for Aquaculture [J]. Transactions of the Chinese Society for Agricultural Machinery, 2011, 42(9):191-196

[3] Li Hui, Liu Xingqiao, Li Jing, et al. Aquiculture remote monitoring system based on IOT Android platform [J]. Transactions of the Chinese Society of Agricultural Engineering, 2013, 29(13): $175-181$.

[4] Qian Zhi-hong,Wang Yi-jun. Internet of Things-oriented Wireless Sensor Networks Review [J]. Journal of Electronics \& Information Technology, 2013, 35(1):215-227

[5] Li Hua, Li Wenxiu. Wireless sensory networks for the livestock, poultry and aquaculture industry pollution monitoring applications [J]. Fishery Modernization, 2008, 35(2):28-31

[6] Jiang Yanliang. Remote control system based on Android intelligent terminal [J]. Application of Electronic Technique, 2012, 38(8):129-132 